\title{
Nota sobre o comportamento de agregação dos machos de Oxaea austera Gerstaecker (Hymenoptera, Apoidea, Oxaeinae) na caatinga do Estado da Bahia, Brasil ${ }^{1}$
}

\author{
Favízia Freitas de Oliveira ${ }^{2}$ \\ Marina Siqueira de Castro $^{3}$
}

\begin{abstract}
Notes on a male sleeping aggregation behavior of Oxaea austera Gerstaecker (Hymenoptera, Apoidea, Oxaeinae) in the caatinga of Bahia State, Brazil. This note reports for the first time a "male sleeping aggregation" of the solitary bee Oxaea austera Gerstaecker, 1867 (Hymenoptera, Apoidea, Oxaeinae) found near the town of Iaçú, Bahia, in Northeastern Brazil. This is also the first record of a species of Oxaea for the caatinga ecosystem.
\end{abstract}

KEY WORDS. Oxaea austera, solitary bee, male sleeping aggregation, caatinga, Brazil

Em uma das expedições para estudo das abelhas da caatinga, realizada na Fazenda Nova América, Município de Iaçú (1246'S e 40¹3'W), Bahia, em janeiro de 2000, teve-se a oportunidade de observar pela primeira vez uma agregação de machos de Oxaea austera Gerstaecker, 1867 (Hymenoptera, Apoidea, Oxaeinae), para "passarem a noite".

Este tipo de comportamento, denominado "male sleeping aggregation", foi descrito por EVANS \& LiNSLEY (1960), LINSLEY \& MiCHENER (1962), CAZIER \& LINSLEY (1963), LINSLEY \& CAZIER (1972) e HURD \& LINSLEY (1976), para outros Oxaeinae do gênero Protoxaea (Protoxaea gloriosa (Fox 1893), espécie da América do Norte). LINSLEY (1962) fornece dados sobre "sleeping aggregations" (incluindo "male sleeping aggregation") para outros Hymenoptera (Aculeata) da América do Norte, a exemplo de abelhas dos gêneros Caupolicana Spinola, 1851 (Colletidae), Anthidiellum Cockerell, 1904, Coelioxys Latreille, 1809 (Megachilidae), Anthophora Latreille, 1803, Melissodes Latreille, 1829, Triepeolus Robertson, 1901, Xeromelecta Linsley, 1939 (Anthophoridae), Agapostemon Guérin-Méneville, 1844 e Lasioglossum Curtis, 1833 (Halictidae), este último gênero estudado também por MIYANAGA \& MAETA (1998). Outros insetos, a exemplo das borboletas do gênero Heliconius Kluk, 1802 apresentam comportamento semelhante, descrito por diversos autores incluindo MALLET (1986). Os critérios iniciais para escolha dos sítios de agregação ainda são desconhecidos. EvANS \& LINSLEY (1960) sugerem que este tipo de comportamento pode ter uma função de proteção, mas que a

1) Contribuição número 1340 do Departamento de Zoologia, Universidade Federal do Paraná.

2) Departamento de Zoologia, Universidade Federal do Paraná. Caixa Postal 19020, 81531-990 Curitiba, Paraná, Brasil. E-mail: favos@bol.com.br

3) Laboratório de Abelhas, Empresa Baiana de Desenvolvimento Agrícola. Avenida Ademar de Barros 967, Ondina, 40170-110 Salvador, Bahia, Brasil. E-mail: marina@ e-net.com.br

Revta bras. Zool. 19 (1): $301-303,2002$ 
concentração antecipada, no momento em que eles começam a se agregar, não os protege de todos os predadores, visto que estes podem tirar vantagem da agregação para se alimentarem.

ZANELLA (2000) relata a não ocorrência do gênero Oxaea Klug, 1807 na caatinga. A presente comunicação científica tem como objetivo principal, notificar o fenômeno de "agregação noturna dos machos" de Oxaea austera, bem como a ocorrência desta espécie na caatinga baiana, fornecendo informações complementares para o conhecimento da bionomia e distribuição geográfica dos Oxaeinae.

A agregação de machos de $O$. austera encontrava-se na extremidade de um dos galhos da espécie arbórea Cammiphora leptophloeos (Mart.) Gillet (Burseraceae), muito comum na região e conhecida popularmente como "umburana de cambão". A referida árvore encontrava-se na borda de um "lajedo", e o galho onde se encontravam as abelhas estava também dirigido para este mesmo lajedo. A congregação localizava-se a uma distância aproximada de dois metros em relação ao solo (lajedo). O fenômeno foi observado na estação chuvosa, quando a vegetação encontra-se bastante verde. Embora com abundante folhagem, a árvore não estava florida.

O aglomerado formava uma estrutura esférica com diâmetro de aproximadamente $15 \mathrm{~cm}$, incluindo o diâmetro do galho $(5 \mathrm{~cm})$. A formação não era fixa: alguns machos saíam da mesma, voando ao redor da árvore, e seus lugares eram imediatamente ocupados por outros machos; posteriormente os que se dispersavam retornavam para a formação. As abelhas ficavam meio superpostas (de maneira desorganizada), por entre as poucas folhas da extremidade do galho, e próximas ao ramo principal, com as pernas estendidas e apoiadas nas folhas (embora em movimento dentro da agregação). Em algumas espécies dos gêneros estudados, pelos autores anteriormente citados, as abelhas se fixavam aos galhos das árvores utilizando as mandíbulas, porém no caso de $O$. austera este comportamento não foi evidenciado, talvez devido ao fato de que a aglomeração foi observada no início de sua formação, o que explica a dispersão de alguns machos e o seu posterior retorno à formação, bem como a aparente desorganização da agregação. Não se evidenciou substância alguma sobre o galho, nem cheiro algum que justificasse a aglomeração dessas abelhas. HURD \& LinSLEY (1976) afirmam que depois que os machos de Protoxaea gloriosa selecionam o local de congregação, este é marcado com excremento amarelo de odor notavelmente perceptível.

O fenômeno de agregação de $O$. austera foi observado em torno das 17 horas, e os machos chegavam aos poucos à formação. Segundo trabalhos que relatam este tipo de comportamento (e.g. HURD \& LINSLEY 1976), provavelmente os machos aglomeravam-se para dormir, visto que se aproximava o pôr do sol. De acordo com estes autores, os machos são fortemente anti-sociais durante o período do dia em que estão tentando estabelecer ou ocupando territórios e se tornam altamente sociáveis quando congregados para passarem a noite.

As abelhas presentes na agregação foram coletadas (25 machos). Dentre estas, duas foram doadas à Coleção de Entomologia J.S. Moure, Departamento de Zoologia, Universidade Federal do Paraná (DZUP), Curitiba, Paraná; duas ao Dr. J.S. Moure e quatro ao Dr. Vinalto Graf (ambos pesquisadores do Departamento de 
Zoologia da Universidade Federal do Paraná); as demais $(\mathrm{n}=17)$, permaneceram na Coleção Regional Moure \& Costa, Laboratório de Abelhas, Empresa Baiana de Desenvolvimento Agrícola (LABE - EBDA), Salvador, Bahia.

\begin{abstract}
AGRADECIMENTOS: A José Xavier de Jesus (técnico da EBDA) e Emilson Cerqueira, que nos auxiliaram nesta expedição. Aos professores Jesus Santiago Moure e Vinalto Graf (Universidade Federal do Paraná), pela identificação das abelhas. À Empresa Baiana de Desenvolvimento Agrícola (EBDA) pelo financiamento da expedição. À CAPES pela concessão da Bolsa de Doutorado para a primeira autora.
\end{abstract}

\title{
REFERÊNCIAS BIBLIOGRÁFICAS
}

CAzier, M.A. \& E.G. Linsley. 1963. Territorial Behavior among Males of Protoxaea gloriosa (Fox) (Hymenoptera: Andrenidae). Can. Ent. 95: 547-556.

Evans, H. E. \& E. G. Linsley. 1960. Notes on the Sleeping Aggregation of Solitary Bees and Wasp. Bull. South. California Acad. Sci. 59: 30-37.

HURD JR., P.D. \& E.G. LinsLeY. 1976. The Bee Family Oxaeidae with a Revision of the North American Species (Hymenoptera: Apoidea). Smith. Contrib. Zool. 220: 1-75.

LinSLEY, E.G. 1962. Sleeping Aggregations of Aculeate Hymenoptera - II. Ann. Ent. Soc. Amer. 55: 148-164.

Linsley, E.G. \& C.D. Michener. 1962. Brief notes on the habits of Protoxaea gloriosa (Hymenoptera, Andrenidae). Jour. Kansas Ent. Soc. 35: 385-389.

Linsley, E.G. \& M.A. CAZIER. 1972. Diurnal and Seasonal Behavior Patterns Among Adults of Protoxaea gloriosa (Hymenoptera, Oxaeidae). Amer. Mus. Novit. 2509: 1-25.

Mallet, J. 1986. Gregarious Roosting and Home Range in Heliconius Butterflies. Nat. Geogr. Res. 2 (2): 198-205.

MryanAGA, R. \& Y. MAETA. 1998. Notes on a male sleeping aggregation of Lasioglossum (Ctenonomia) kumejimense (Hymenoptera: Halictidae). Jour. Ent. Sci. 1 (3): 357-358.

Zanella, F.C.V. 2000. The Bees of the Caatinga (Hymenoptera, Apoidea, Apiformes): a species list and comparative notes regarding their distribution. Apidologie 31: 579-592.

Recebido em 04.V.2001; aceito em 18.II.2002. 